\title{
Evaluation of microvascular findings of deeply invasive colorectal cancer by endocytoscopy with narrow-band imaging
}

Authors

Institutions
Hiroki Nakamura', Shin-ei Kudo' ${ }^{1}$, Masashi Misawa', Shinichi Kataoka', Kunihiko Wakamura', Takemasa Hayashi', Toyoki Kudo ${ }^{1}$, Yuichi Mori ${ }^{1}$, Kenichi Takeda ${ }^{1}$, Katsuro Ichimasa' ${ }^{1}$, Hideyuki Miyachi' ${ }^{1}$, Atushi Katagiri ${ }^{1}$, Fumio Ishida ${ }^{1}$, Haruhiro Inoue ${ }^{2}$

${ }^{1}$ Digestive Disease Center, Showa University, Northern Yokohama Hospital, Yokohama, Japan

${ }^{2}$ Digestive Disease Center, Showa University, Koto-Toyosu Hospital, Tokyo, Japan submitted 1. April 2016 accepted after revision 13. September 2016

\section{Bibliography}

Dol http://dx.doi.org/

10.1055/s-0042-117629

Published online: 10.11.2016

Endoscopy International Open 2016; 04: E1280-E1285

(c) Georg Thieme Verlag KG

Stuttgart · New York

E-ISSN 2196-9736

\section{Corresponding author}

\section{Hiroki Nakamura}

Showa-University Northern

Yokohama Hospital -

Digestive Disease Center

35-1,Chigasaki-chu-o Tsuduki

Yokohama Kanagawa 2240032

Japan

Phone: +08109080359853

Fax: 0459497738

n-hiroki@td6.so-net.ne.jp
Background and study aims: Magnifying narrowband imaging (NBI) is useful for examination of colorectal lesions, and endocytoscopy (EC) allows diagnostic evaluation of structural atypia, nuclear atypia, and vascular structures of colorectal tumors. The aim of this study was to examine surface microvessels in deep invasive colorectal cancer using EC with a new NBI video processor system.

Patients and methods: We retrospectively assessed 132 colorectal neoplastic lesions: 81 adenomas, 18 intramucosal cancers, 4 submucosal slightly invasive cancers, and 29 submucosal deep invasive cancers. Detailed vascular findings commonly seen in submucosal deep invasive carcinomas included $>2$-fold vasodilatation seen in adenomas, abnormal tortuosity and branching,

\section{Introduction}

$\nabla$

The development of endoscopy has greatly benefited diagnosis of colorectal neoplasia. Chromoendoscopy has been used to establish the pit pattern classification, which is effective for differentiation between non-neoplasia and neoplasia as well as between adenoma and cancer [1]. Narrow-band imaging (NBI) was developed in 2006; this technique uses superficial tissue structures and emphasizes the imaging of certain features [2], such as vascular and mucosal patterns. Endocytoscopy (EC) was more recently developed and can magnify objects by 380 -fold to 450 -fold. EC enables on-site observation of structural and nuclear irregularities [3,4], and therefore has the potential to allow for "optical biopsy" [5]. The qualitative and quantitative usefulness of superficial microvascular findings in the examination of colorectal lesions with NBI magnification endoscopy (NBI$\mathrm{ME}$ ) has been reported by many institutions [69], but the utility of EC with NBI (EC-NBI) has not been demonstrated. loss of the micro-network pattern, caliber change in $>2$ places in a single blood vessel, and blood vessels not visible in a line because they appear like a string of beads (beaded sign).

Results: Univariate analysis revealed 4 vascular findings that were strongly predictive of submucosal deep invasion: vasodilatation (odds ratio [OR] 9.31; 95\% confidence interval [CI] 3.5724.30), loss of the micro-network pattern (OR 61.60; 95\% CI 17.87 - 212.29), caliber change (OR 35.7; 95\% CI 9.16-139.14), and the beaded sign (OR 45.90; 95\% CI 5.50-382.73).

Conclusions: Detailed assessment of ultra-magnified microvessels could improve the diagnostic performance for submucosal deep invasive cancer.

Study registration: UMIN-CTR000014033

Kudo et al. [10] classified the endocytoscopic vascular pattern (EC-V) into 3 types ( $\bullet$ Fig. 1 ): obscure surface microvessels (EC-V1), clearly observed surface microvessels of a uniform caliber and arrangement (EC-V2), and dilated surface microvessels of a non-homogeneous caliber or arrangement (EC-V3). EC-V1 mainly corresponds to non-neoplasia, EC-V2 to neoplasia, and EC-V3 to invasive cancer. However, whether the vascular findings of EC-V3 are irregularities remains unclear.

The aims of this study were to examine the vascular findings of submucosal deep invasive cancer by EC-NBI with a new video processor system (EVIS LUCERA ELITE SYSTEM; Olympus, Tokyo, Japan) and to determine the clinical significance of these ultra-high magnification findings.

\section{License terms}

()(1) $\Theta \circledast$ 

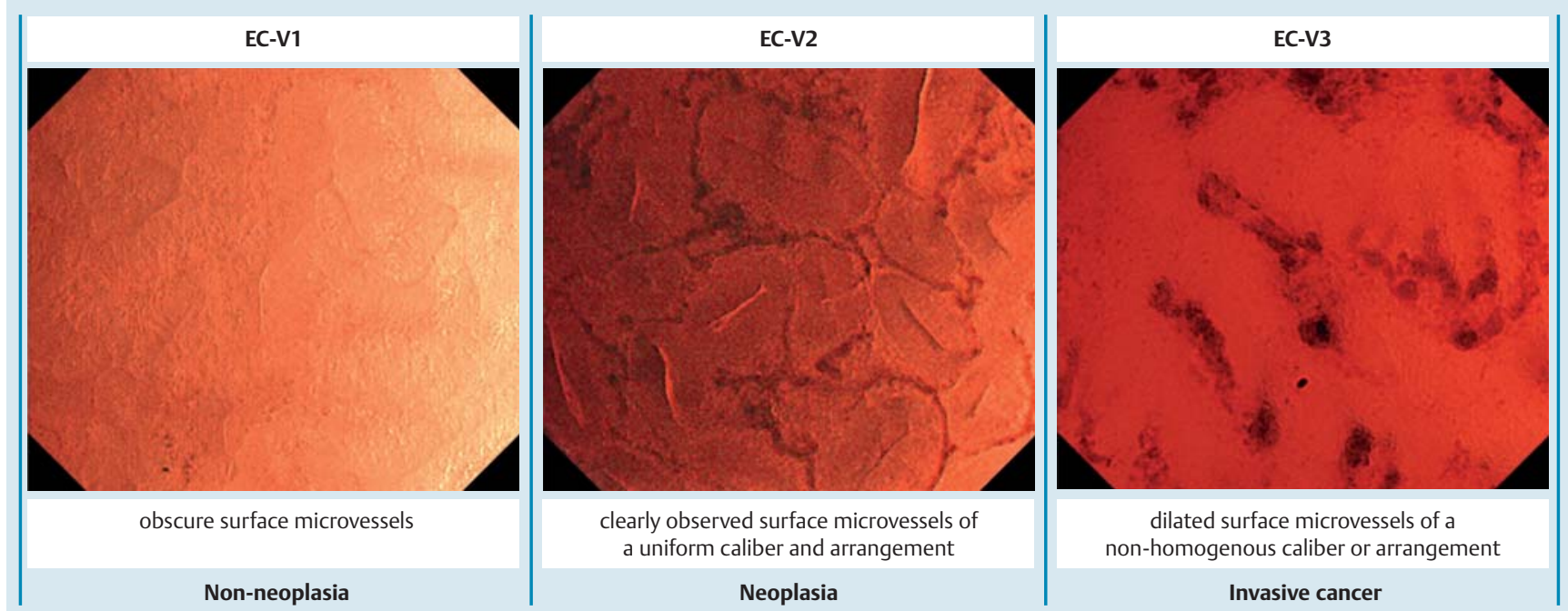

Fig. 1 Endocytoscopic vascular pattern (EC-V) classification.

\section{Patients and methods}

\section{Patients}

We retrospectively analyzed 98 patients who underwent endoscopic or surgical resection after observation with EC-NBI from May 2013 to December 2014. We excluded patients with inflammatory bowel disease and those with hyperplastic polyps (including sessile serrated adenomas/polyps) and cancers deeper than T2. Before the examination, patients underwent bowel preparation with $2 \mathrm{~L}$ to $3 \mathrm{~L}$ of polyethylene glycol solution. Diazepam and butylscopolamine were used intravenously for sedation and prevention of peristalsis. The study took place at the Digestive Disease Center of Showa University Northern Yokohama Hospital. The protocol was approved by the Medical Ethics Committee of our hospital (No.1405-03; approved on June 6, 2014) and registered in the University Hospital Medical Information Network Clinical Trials Registry (UMIN-CTR000014033; approved on May 25, 2014). All participants gave written informed consent, and the study was conducted according to the Declaration of Helsinki.

\section{EC system}

The endocytoscope (CF-Y0020; Olympus) had a magnification range of 80 to $380 \times$ with a standard video processor system (EVIS LUCERA ELITE SYSTEM; CV-290/CLV-290SL) and a digital image filing system (Solemio; Olympus). The EC-NBI was set at enhancement mode A8 and color mode 3. The endocytoscope had a working length of $133 \mathrm{~cm}$, outer diameter of $13.6 \mathrm{~mm}$, and single lens. The instrument allowed gradual magnification at the center of the monitor, thus pinpointing the target area being viewed. Our observation focused on the area of interest, which showed irregularity after conventional NBI magnification before staining. Next, we checked the superficial layers of polyps as thoroughly as possible.

\section{Evaluation of EC-NBI findings}

We anticipated 5 different types of vascular findings in light of the detailed vascular findings commonly seen in submucosal deep invasive cancer: 1) caliber dilatation; 2) abnormal tortuosity and branching of microvessels; 3 ) loss of the micro-network (MN) pattern; 4) caliber change; and 5) beaded sign. First, we de-

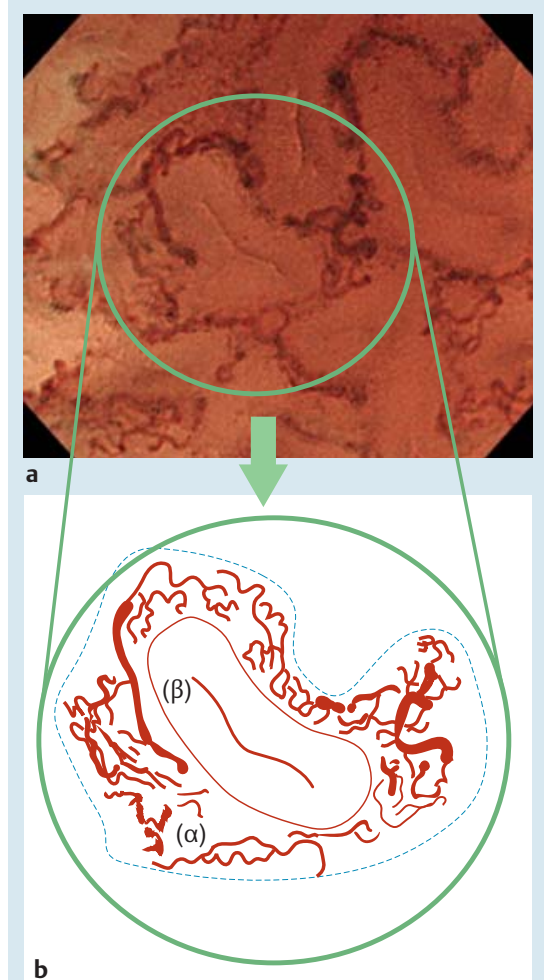

Fig. 2 Loss of the micro-network (MN) pattern. a Image of typical adenoma. b Schema of MN pattern; typical adenoma has fine and tortuous vessels in the intervening part ( $\alpha$ in dotted line) around the crypt opening $(\beta)$. We defined these vessels as the MN pattern. c Image of submucosal deep invasive cancer; loss of the MN pattern.

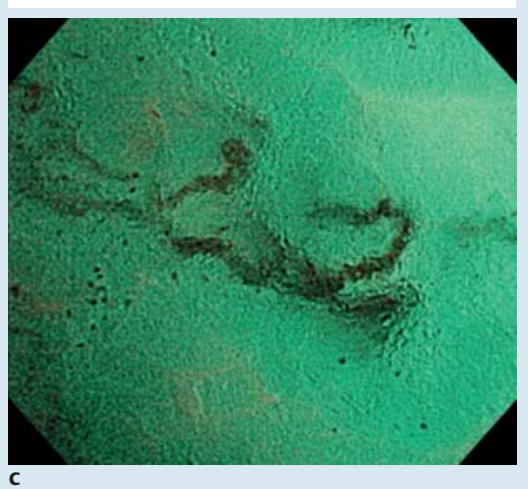




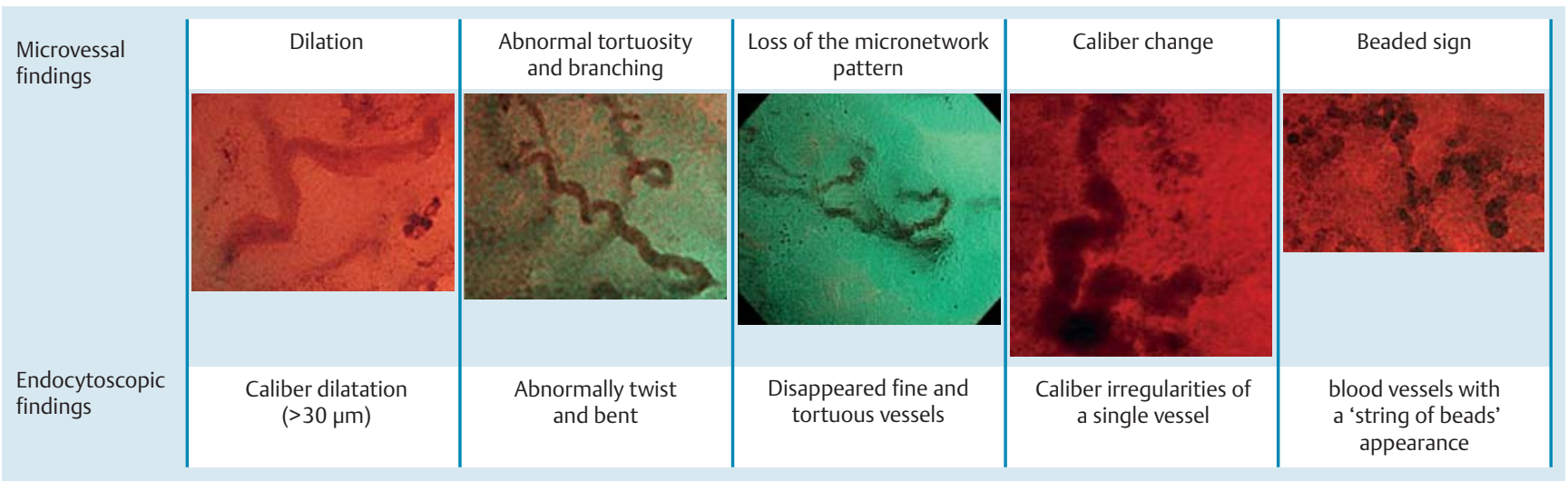

Fig. 3 Microvascular findings and schema.

fined caliber dilation as vessel thickening $>2$-fold $(>30 \mu \mathrm{m})$ that seen in adenomas. We reported a mean blood vessel caliber size measured by EC of $19.5 \pm 4.2 \mu \mathrm{m}$ in adenoma and $33.0 \pm 7.2 \mu \mathrm{m}$ in T1 cancer [11]. We therefore set a threshold caliber of $30 \mu \mathrm{m}$, which was almost twice that for adenoma vessels, to allow us to detect caliber dilation. As for abnormal tortuosity and branching of microvessels, vessels are usually arranged along the crypt opening in adenoma. Abnormal tortuosity and branching was defined as sharply angled microvessels or thick, irregular vessels passing from a deep layer to join a surface vessel. We defined the MN pattern as the presence of fine and tortuous vessels in the intervening part around the crypt opening of adenomas [12] ( $\bullet$ Fig. 2). However, the MN pattern tended to disappear in invasive carcinomas; thus, we regarded loss of the MN pattern as suggestive of invasive carcinoma. Caliber change was defined as a maximum caliber size of at least double the minimum caliber size in 1 vessel. The vascular thickness is relatively uniform in adenomas, but seemed to become less uniform in cases of submucosal deep invasive cancer. The beaded sign was defined as blood vessels with the appearance of a string of beads ( $\bullet$ Fig. 3 ). The blood vessel diameter was measured using Nitenkeisokuki version 1.1.1. and was evaluated relative to an endoscopic screen width of $600 \mu \mathrm{m}$. Endoscopic images were randomly allocated to 2 readers (H.N. and M.M.) for evaluation without a final pathologic diagnosis.

\section{NBI-ME classification}

We classified NBI-ME findings into 6 categories according to the vascular pattern classification [9]. Sparse and irregular patterns were considered the index of submucosal deep invasive cancer.

\section{Outcome measure and statistical analysis}

We calculated the sensitivity, specificity, and overall accuracy of each endoscopic modality. Furthermore, we compared the diagnostic accuracy between the EC-V and NBI-ME. We used SPSS for Windows version 20.0 (IBM Corp., Armonk, NY, USA) for data analysis. Pearson's chi-square test was applied to calculate the odds ratios (ORs) of the endocytoscopic findings, and McNemar's test was applied to compare the diagnostic accuracy between EC-V and NBI-ME. $\mathrm{K}$ values were calculated for interobserver agreement. A $\mathrm{K}$ value of 0.00 indicated poor agreement; 0.00 0.20 , slight agreement; $0.21-0.40$, fair agreement; $0.41-0.60$, moderate agreement; $0.61-0.80$, substantial agreement; and $0.80-1.00$, almost perfect agreement. $P$-values of $<0.05$ were considered statistically significant.

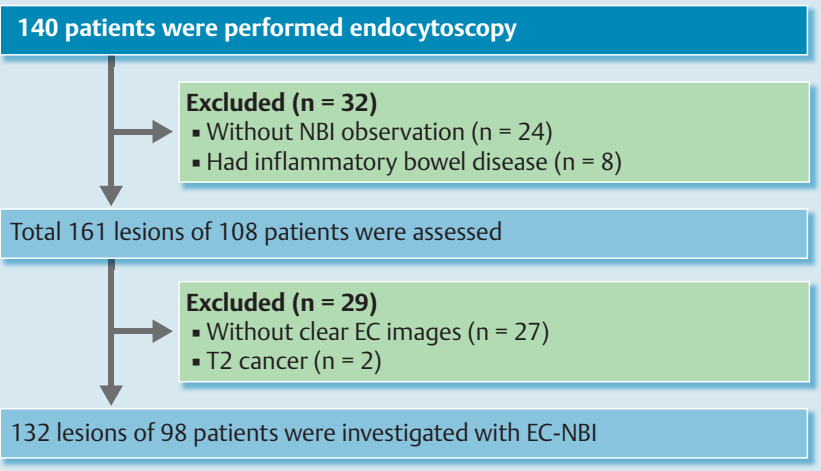

Fig. 4 Patient flowchart.

\section{Pathologic diagnosis}

All specimens were fixed in $10 \%$ buffered formalin solution after retrieval. They were stained with conventional hematoxylin and eosin and diagnosed using the JSCCR guideline for the degree of submucosal invasion; each cancer was subclassified accordingly [13].

\section{Results}

Overall, 163 lesions were included. Of these, 31 lesions were excluded for various reasons ( $\bullet$ Fig.4). We therefore assessed 132 lesions: 81 adenomas, 18 intramucosal cancers, 4 submucosal slightly invasive cancers, and 29 submucosal deep invasive cancers. In total, 959 pictures of these lesions were evaluated. Patient demographics and lesion characteristics are summarized in Table 1. Sensitivity, specificity, and diagnostic accuracy of the caliber dilation for differentiating adenoma/intramucosal cancer/ submucosal slightly invasive cancer or submucosal deep invasive cancer were $75.9 \%, 74.8 \%$, and $75.0 \%$, respectively $(P<0.01$; OR 9.31). Furthermore, sensitivity, specificity, and diagnostic accuracy of abnormal tortuosity and branching were $17.2 \%, 92.2 \%$, and $75.8 \%$, respectively $(P=0.153$; OR 2.47$)$. Sensitivity, specificity, and diagnostic accuracy of loss of the MN pattern were $75.9 \%$, $95.1 \%$, and $90.9 \%$, respectively $(P<0.01$; OR 61.64). Sensitivity, specificity, and diagnostic accuracy of the caliber change were $35.7 \%, 97.1 \%$, and $87.1 \%$, respectively $(P<0.01$; OR 45.78$)$. Sensitivity, specificity, and diagnostic accuracy of the beaded sign 


\begin{tabular}{|c|c|c|c|c|}
\hline & $\begin{array}{l}\text { Adenoma } \\
(n=81)\end{array}$ & $\begin{array}{l}\text { Intramucosal } \\
\text { carcinoma } \\
(n=18)\end{array}$ & $\begin{array}{l}\text { Submucosal slightly } \\
\text { invasive carcinoma } \\
(n=4)\end{array}$ & $\begin{array}{l}\text { Invasive } \\
\text { carcinoma } \\
(\mathbf{n = 2 9})\end{array}$ \\
\hline Age, y & $\begin{array}{l}63.9 \pm 13.1 \\
(32-91)\end{array}$ & $\begin{array}{l}66.5 \pm 8.7 \\
(50-81)\end{array}$ & $\begin{array}{l}71.8 \pm 3.3 \\
(68-75)\end{array}$ & $\begin{array}{l}63.2 \pm 13.5 \\
(36-94)\end{array}$ \\
\hline \multicolumn{5}{|l|}{ Sex } \\
\hline Male & 58 & 10 & 4 & 16 \\
\hline Female & 23 & 8 & 0 & 13 \\
\hline \multicolumn{5}{|l|}{ Location } \\
\hline Left side of colon & 41 & 5 & 1 & 7 \\
\hline Right side of colon & 30 & 8 & 1 & 12 \\
\hline Rectum & 10 & 5 & 2 & 10 \\
\hline Size of lesions, mm & $\begin{array}{l}10.7 \pm 6.4 \\
(3-35)\end{array}$ & $\begin{array}{l}18.7 \pm 10.0 \\
(4-40)\end{array}$ & $\begin{array}{l}18.5 \pm 9.9 \\
(4-25)\end{array}$ & $\begin{array}{l}20.4 \pm 7.1 \\
(8-35)\end{array}$ \\
\hline Protruded and flat-elevated type & 77 & 14 & 2 & 21 \\
\hline Depressed type & 4 & 4 & 2 & 8 \\
\hline
\end{tabular}

Data are presented as mean \pm standard deviation (range) or $\mathrm{n}$.

\begin{tabular}{|llllll|}
\hline Vascular findings & Sensitivity & Specificity & Accuracy & $\begin{array}{l}\text { OR } \\
\text { (95\% CI), \% }\end{array}$ & P value \\
\hline Caliber dilation & & & & 75.01 \\
\hline Abnormal tortuosity and branching & $75.9 \%$ & $74.8 \%$ & $75.0 \%$ & 9.31 & $<0.01$ \\
& $(22 / 29)$ & $(77 / 103)$ & $(99 / 132)$ & $(3.57-24.3)$ & \\
\hline Loss of the micro-network pattern & $17.2 \%$ & $92.2 \%$ & $75.8 \%$ & 2.47 & 0.153 \\
\hline Caliber change & $(5 / 29)$ & $(95 / 103)$ & $(100 / 132)$ & $(0.74-8.25)$ & \\
\hline & $(22 / 29)$ & $(98 / 103)$ & $(120 / 132)$ & $(17.87-212.29)$ & $<0.01$ \\
\hline Beaded sign & $35.7 \%$ & $97.1 \%$ & $87.1 \%$ & 45.78 & $<0.01$ \\
& $(15 / 29)$ & $(100 / 103)$ & $(115 / 132)$ & $(9.16-139.14)$ & \\
\hline
\end{tabular}

Table 2 Diagnostic accuracy of microvascular findings.

Total number of polyps: 132 .

$\mathrm{Cl}$, confidence interval; OR, odds ratio.

Table 3 Comparison of diagnostic accuracy between EC-V and NBI-ME.

\begin{tabular}{|llll|}
\hline Predicting invasive cancer (T1b) & EC-V3 & NBI-ME & P value \\
\hline Sensitivity & $96.3 \%$ & $82.1 \%$ & 0.375 \\
\hline Specificity & $97.1 \%$ & $94.2 \%$ & 0.219 \\
\hline Accuracy & $97.0 \%$ & $91.7 \%$ & 0.065 \\
\hline
\end{tabular}

McNemar's test was applied.

EC-V, endocytoscopic vascular pattern; NBI-ME, NBI magnification endoscopy.

were $31.0 \%, 99.0 \%$, and $84.1 \%$, respectively $(P<0.01$; OR 45.95$)$ ( Table 2).

The interobserver agreement $\mathrm{K}$ value between experienced endoscopists for dilation was 0.67 , that for abnormal tortuosity and branching was 0.44 , that for loss of the MN pattern was 0.55 , that for caliber change was 0.52 , and that for the beaded sign was 0.55 .

Sensitivity, specificity, and overall accuracy of EC-NBI were $96.3 \%, 97.1 \%$, and $97.0 \%$, respectively, among the cases included in this study. On the other hand, NBI-ME had a sensitivity of $82.1 \%$, specificity of $94.2 \%$, and overall accuracy of $91.7 \%$ (๑ Table 3).

\section{Discussion}

Our results suggest that caliber dilation, loss of the MN pattern, caliber change, and the beaded sign are highly correlated with submucosal deep invasive cancers. In addition, the endoscopists showed moderate agreement, and the results obtained were validated.

With recent advances in endoscopic techniques such as endoscopic submucosal dissection, even a large lesion requiring endoscopic piecemeal mucosal resection can be resected en bloc. It has also become possible to resect non-lifting lesions other than submucosal deep invasive cancer, because of fibrosis, after biopsy or injection of liquid, and other circumstances. For appropriate treatment, however, it is important to differentiate whether the lesion is submucosal slightly invasive cancer or submucosal deep invasive cancer. Therefore, high specificity is required to prevent excessive surgical treatment. In a comparison of diagnostic accuracy between EC-V and NBI-ME, all of these diagnostic parameters were higher with EC-V than with NBI-ME. However, there were no significant differences between EC-NBI and NBI$\mathrm{ME}$ in terms of these parameters. However, all cases evaluated as EC-V3 and as having an irregular/sparse pattern of NBI-ME were classified as submucosal deep invasive cancer. This finding seems to illustrate an advantage of EC-NBI.

EC-NBI can allow for observation of vascular irregularity in vivo and might facilitate observation of angiogenesis of colorectal lesions [11]. Konerding et al. [14] described the normal colonic mucosa as having honeycomb-like vasculature. Colorectal tumor capillaries become thick due to angiogenesis, the caliber changes as the grade of atypia increases, the vessels become blocked, and changes in vascular density occur [15]. Ultra-high magnification of EC-NBI may enable the blood vessels to be observed in a manner close to these models. EC-NBI has shown that tumor capillaries are thicker than non-tumor capillaries and that the capillaries 

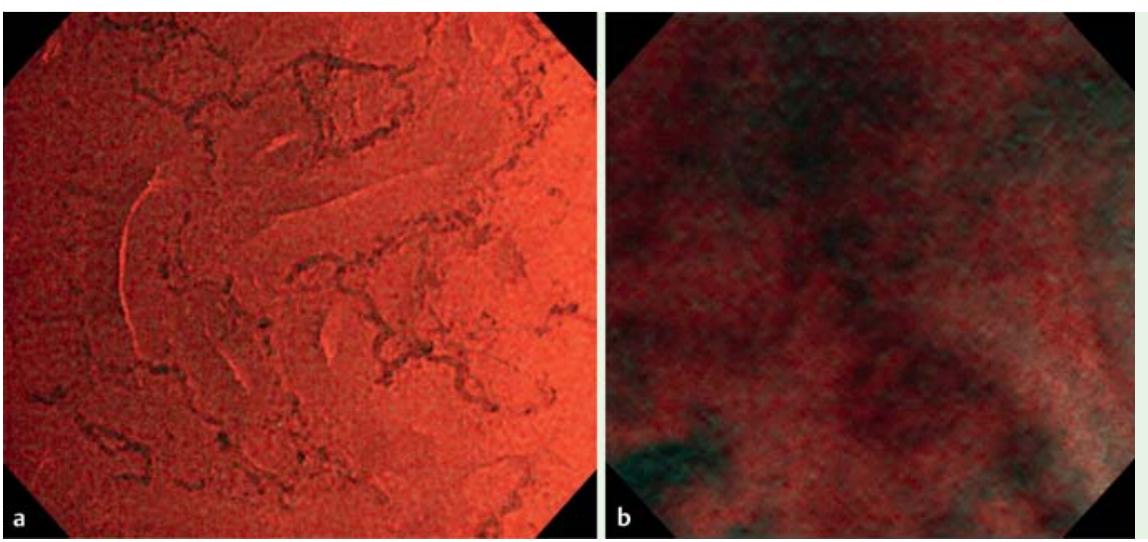

Fig. 5 Comparison of endocytoscopy with narrowband imaging using EVIS LUCERA ELITE SYSTEM and EVIS LUCERA SPECTRUM SYSTEM. The (a) EVIS LUCERA ELITE SYSTEM images became brighter and clearer than the (b) EVIS LUCERA SPECTRUM SYSTEM images.

are significantly thick in cases of submucosal deep invasive cancer. Loss of the MN pattern is considered to reflect a phenomenon of decreasing vascular density, although severely atypical blood vessels remain as the lesion develops into submucosal deeply invasive cancer. Although significant differences were observed in vascular dilation, the diagnostic accuracy was $75.6 \%$. This result was lower than that in other cases. A previous study that utilized microvascular corrosion casting revealed that invasive cancer has significantly larger microvessel diameters [14]. The vascular dilation could therefore be the most important finding in submucosal deep invasive cancer. Conversely, there were no significant differences in tortuosity. In NBI-ME, tortuosity is an important abnormal vascular finding, and its significance has been confirmed. However, at $380 \times$ magnification with EC, even usual adenomas show mild tortuosity. It becomes difficult to distinguish normal from abnormal findings according to tortuosity.

When we evaluated the detailed vascular findings in this study, high-quality images were needed. We used the EVIS LUCERA SPECTRUM SYSTEM until 2012. With this system, the quality of images was usually poor because sufficient brightness was not obtained with EC-NBI. With the advent of the EVIS LUCERA ELITE SYSTEM in October 2012, the images obtained with EC-NBI were brighter and clearer ( $\bullet$ Fig.5). This may have been attributable to increased light intensity, increased exposure time of NBI, and improved noise reduction, thereby allowing clearer observation of the vessels and detailed examination of EC-V. With regard to advantages of EC-NBI over conventional EC, conventional EC always requires staining with crystal violet and methylene blue $[16,17]$. However, EC-NBI does not require staining, and testing with EC-NBI therefore can be performed safely and quickly. We recently reported on use of a computer-aided diagnosis (CAD) system for EC-V [18]. The CAD system provided sufficient diagnostic ability for lesion characterization, but it could not predict the invasion depth of colorectal cancer. Therefore, EC-V still has an advantage over the CAD system.

Future development of quantitative and qualitative diagnostic techniques will use elements different from those of EC classification because EC-NBI does not focus on the nucleus and gland formation of surface colorectal lesions but on the vascular caliber and its irregularity. For example, when cancer is deep-seated and not exposed to the surface, nuclear or structural atypia may not be seen by EC. In these cases, vascular findings might help to diagnose the submucosal deeply invasive area. Furthermore, it might be possible to predict potential metastasis, such as venous invasion, by comparing vascular irregularities and thus select candidates for vascular endothelial growth factor inhibitors (e.g., bevacizumab) or predict treatment efficacy $[19,20]$.
This study had some limitations. First, it was retrospective study and conducted in a single institution. We are planning to increase the number of patients for a further prospective study. Second, although EC enables the living body to be observed, it is difficult to compare the results with pathologic results. We considered that because the focal depth of the endocytoscope is about $50 \mu \mathrm{m}$, comparison with dilated vessels on the surface would be possible. However, it was actually difficult to evaluate deformed blood vessels, including changes in vascular caliber, because of dehydration and staining during specimen preparation. Therefore, it may be necessary to consider that pathologic findings are new diagnostic findings in the living body.

\section{Conclusion}

\section{$\nabla$}

In conclusion, dilation, loss of the MN pattern, caliber change, and a beaded sign are important vascular findings observed by EC in patients with colorectal submucosal deep invasion. These results appear to contribute to the definition of irregular vascular findings of EC-V3.

\section{Competing interests: None}

\section{References}

1 Kudo S, Hirota S, Nakajima T et al. Colorectal tumours and pit pattern. J Clin Pathol 1994; 47: 880-885

2 Gono K, Obi T, Yamaguchi $M$ et al. Appearance of enhanced tissue features in narrow-band endoscopic imaging. J Biomed 2004; 9: 568 - 577

3 Haruhiro I, Kudo S-E, Shiokawa A et al. Observation of Living Cancer Cells in the Gastrointestinal Tract. Clin Gastroenterol Hepatol 2005; 3565: $61-63$

4 Kudo SE, Wakamura K, Ikehara $N$ et al. Diagnosis of colorectal lesions with a novel endocytoscopic classification a pilot study. Endoscopy 2011; 43: 869-875

5 Mori $Y$, Kudo S, Ikehara $N$ et al. Comprehensive diagnostic ability of endocytoscopy compared with biopsy for colorectal neoplasms: A prospective randomized noninferiority trial. Endoscopy 2013; 45: 98 105

6 Sano Y, Horimatsu T, Fu KI et al. Magnifying observation of microvascular architecture of colorectal lesions using a narrow-band imaging system. Dig Endosc 2006; 18: 44-51

7 Katagiri A, Fu KI, Sano Y et al. Narrow band imaging with magnifying colonoscopy as diagnostic tool for predicting histology of early colorectal neoplasia. Aliment Pharmacol Ther 2008; 27: 1269-1274

8 Hirata M, Tanaka S, Oka S et al. Magnifying endoscopy with narrow band imaging for diagnosis of colorectal tumors. Gastrointest Endosc 2007; 65: 988 -995

9 Wada Y, Kudo SE, Kashida $H$ et al. Diagnosis of colorectal lesions with the magnifying narrow-band imaging system. Gastrointest Endosc American Society for Gastrointestinal Endoscopy 2009; 70: 522-531 
10 Kudo S, Misawa M, Wada Y et al. Endocytoscopic microvasculature evaluation is a reliable new diagnostic method for colorectal lesions. Gastrointest Endosc 2015; 82: 1 - 12

11 Takeda K, Kudo SE, Misawa M et al. Comparison of the endocytoscopic and clinico-pathologic features of colorectal neoplasms. EIO 2016; 04 : $397-402$

12 Yagi K, Nozawa $Y$, Endou $S$ et al. Diagnosis of early gastric cancer by magnifying endoscopy with NBI from viewpoint of histological imaging: Mucosal patterning in terms of white zone visibility and its relationship to histology. Diagn Ther Endosc 2012; 954809: 1 - 7

13 Watanabe T, Itabashi M, Shimada Yet al., Japanese Society for Cancer of the Colon and Rectum. Japanese Society for Cancer of the Colon and Rectum (JSCCR) Guidelines 2014 for treatment of colorectal cancer.

14 Konerding M, Fait E, Gaumann A. 3D microvascular architecture of precancerous lesions and invasive carcinomas of the colon. Br J Cancer 2001; 84: $1354-1362$
15 Skinner SA, Frydman GM, O'Brien PE. Microvascular structure of benign and malignant tumors of the colon in humans. Dig Dis Sci 1995; 40: $373-384$

16 Sasajima $K$, Kudo SE, Inoue $H$ et al. Real-time in vivo virtual histology of colorectal lesions when using the endocytoscopy system. Gastrointest Endosc 2006; 63: 1010-1017

17 Ichimasa K, Kudo SE, Mori Y et al. Double staining with crystal violet and methylene blue is appropriate for colonic endocytoscopy: An in vivo prospective pilot study. Dig Endosc 2014; 26: 403 - 408

18 Misawa M, Kudo S, Mori Y et al. Characterization of Colorectal Lesions Using a Computer-Aided Diagnostic System for Narrow-Band Imaging Endocytoscopy. Gastroenterology 2016; 150: 1531 - 1532

19 Goel S, Duda DG, Xu L et al. Normalization of the Vasculature for Treatment of Cancer and Other Diseases. Physiol Rev 2011; 91: 1071-1121

20 Jain RK. Normalization of tumor vasculature: an emerging concept in antiangiogenic therapy. Science 2005; 307: 58-62 